\title{
Double Endoscopic Intraluminal Operation (DEILO) for Early Gastric Cancer: Outcome of Novel Procedure for Endoscopic Submucosal Dissection
}

\author{
KYOICHI OGATA ${ }^{1}$, MITSUHIRO YANAI ${ }^{1}$, KENGO KURIYAMA $^{1}$, MASAKI SUZUKI ${ }^{1}$, \\ TORU YANOMA ${ }^{1}$, AKIHARU KIMURA ${ }^{1}$, NORIMICHI KOGURE ${ }^{1}$, YOSHITAKA TOYOMASU ${ }^{2}$, \\ TETSURO OHNO ${ }^{3}$, ERITO MOCHIKI ${ }^{4}$ and HIROYUKI KUWANO ${ }^{1}$ \\ ${ }^{1}$ Department of General Surgical Science, \\ Gunma University Graduate School of Medicine, Maebashi, Japan; \\ ${ }^{2}$ Department of Digestive Surgery, Saitama Cardiovascular and Respiratory Center, Saitama, Japan; \\ ${ }^{3}$ Department of Surgery, Chichibu Hospital, Chichibu, Japan; \\ ${ }^{4}$ Department of Digestive Tract and General Surgery, \\ Saitama Medical Center, Saitama Medical University, Kawagoe, Japan
}

\begin{abstract}
Endoscopic submucosal dissection (ESD) has been used to treat patients with early gastric cancer (EGC). Although several endoscopic devices have been developed to ensure easy and safe ESD, this technique still requires an experienced, highly skilled endoscopist, as it is performed through a single gastroscope, thus requiring one-handed surgical techniques. To overcome these limitations, many ESD procedures with counter-traction have been developed, such as the double scope, double channel scope, clip with line, magnetic anchor, percutaneous traction and external grasping forceps methods. We devised a double endoscopic intraluminal operation (DEILO). Two endoscopes were simultaneously inserted into the stomach. One endoscope was used to lift the lesion, and the other was used to excise the lesion. The DEILO procedure was performed on 122 cases of EGC. In this article, we report the efficacy and safety of DEILO in patients with EGC.
\end{abstract}

Endoscopic submucosal dissection (ESD) has been widely used in Japan and many countries as an alternative to endoscopic mucosal resection (EMR) for the treatment of early gastric cancer (EGC). ESD is the standard treatment for differentiated gastric adenocarcinomas without ulceration, which are clinically

Correspondence to: Kyoichi Ogata, MD, Ph.D., Department of General Surgical Science, Gunma University Graduate School of Medicine 3-39-22 Showa-machi, Maebashi, Gunma 371-8511, Japan. Tel: +81 272208224, Fax: +81 272208230, e-mail: kogata@gunma-u.ac.jp

Key Words: ESD, DEILO, double endoscope, traction, early gastric cancer. diagnosed as T1a (tumor invasion up to the muscularis $e$ mucosae), and have a diameter of $<2 \mathrm{~cm}$ [Japanese Gastric Cancer Treatment Guidelines 2010 (ver. 3) (1)]. In addition, Gotoda et al. evaluated a large database containing information on more than 5,000 patients who had undergone gastrectomy plus lymph node dissection, and determined the risk of lymph node metastasis in different groups of patients with EGC, outlining the need for accurate histological evaluation of resected specimens in order to avoid the recurrence of such lesions (2).

Endoscopic procedures for the resection of EGCs must be safe, effective, and applicable in a wide range of clinical situations. Although several endoscopic devices have been developed to make ESD easier and safer, the technique still requires an experienced, highly skilled endoscopist as it is performed through a single gastroscope and requires onehanded surgical techniques $(3,4)$. Surgeons normally prefer to employ a two-handed technique, as in conventional open and laparoscopic surgery. However, in ESD, the assistant finds it difficult to support the procedure using their left hand, and counter-traction is therefore difficult to achieve. The double endoscopic intraluminal operation (DEILO) was developed to enable the use of two hands. In 2004 and 2010, we reported the application of DEILO to the resection of esophageal and gastric tumors $(5,6)$. The use of two fine endoscopes allows easy dissection of lesions in the lumen of both the esophagus and stomach. Here we report the effectiveness and safety of DEILO in patients with EGC, and review the methods for ESD with traction.

\section{Patients and Methods}

Indications for endoscopic treatment. From 1999 to 2015, at the Department of General Surgical Science, Gunma University Hospital, a total of 122 EGCs treated with DEILO were included in 
Table I. Characteristics of the tumors included in the study.

\begin{tabular}{lc}
\hline & Cases (n=122) \\
\hline Tumor location, n (\%) & \\
Upper third & $19(15.6 \%)$ \\
Middle third & $40(32.8 \%)$ \\
Lower third & $63(51.6 \%)$ \\
Macroscopic type, n (\%)* & \\
Protruded (0-I) & $5(4.1 \%)$ \\
Superficially elevated (0-IIa) & $58(47.5 \%)$ \\
Flat (0-IIb) & $11(9.0 \%)$ \\
Superficially depressed (0-IIc) & $48(39.3 \%)$ \\
Depth of tumor invasion, n (\%) & \\
Mucosa & $102(83.6 \%)$ \\
Submucosa & $20(16.4 \%)$ \\
Histological type, n (\%) & \\
Well-differentiated & $95(77.9 \%)$ \\
Moderately differentiated & $16(13.1 \%)$ \\
Poorly differentiated & $11(9.0 \%)$ \\
Mean tumor size (range), cm & $1.8(0.2-4.2)$ \\
\hline
\end{tabular}

*According to Japanese Classification of Gastric Carcinoma (7).

this study. The clinicopathological characteristics of the 122 tumors are shown in Table I. Of the 122 tumors, only 19 were in the upper third of the stomach. Histopathologic diagnosis showed that the depth of tumor invasion was intramucosal in the majority of tumors, with submucosal infiltration in 20 tumors. One-hundred and eleven tumors were differentiated type adenocarcinoma.

The following standard indications for ESD were proposed by the Japanese Gastric Cancer Association: differentiated adenocarcinoma, intramucosal cancer, lesion $<2 \mathrm{~cm}$, and no ulceration (1). Lesions that meet all these indications are considered to present a negligible risk of lymph node metastasis $(1,2)$. We extended the indications for DEILO, which were based on the treatment guidelines for gastric cancer in Japan, to include the following: intramucosal cancer; differentiated adenocarcinoma, including nonulcerated lesions (regardless of size), or ulcerated lesions $<3 \mathrm{~cm}$; and undifferentiated nonulcerated adenocarcinoma $<2 \mathrm{~cm}$. Therefore, for this study, a surgeon and gastroenterologist performed a preoperative endoscopic assessment on every patient to confirm the histological type based on biopsy specimens and measured the sizes of tumors using forceps. Endoscopic ultrasound was used to determine the depth and size of the tumor, as well as the extent of cancer invasion.

The study was approved by the Institutional Review Board of Gunma University Hospital (approval no. 1263). The aims and methods of the study were fully explained to the participants, and written informed consent was obtained from all patients.

DEILO technique. The DEILO technique has been described in detail previously $(5,6)$. All treatments were performed with the patient under general anesthesia. Airway management consisted of nasal insertion of an endotracheal tube to secure ventilation and to protect against intraoperative aspiration and postoperative pneumonia. Two gastrointestinal endoscopes were inserted orally for DEILO, which allowed a more thorough procedure than diagnostic endoscopy. The first endoscope was a GIF-Q260J (Olympus Co., Ltd., Tokyo, Japan), which has an outer diameter of $9.2 \mathrm{~mm}$ with a disposable attachment

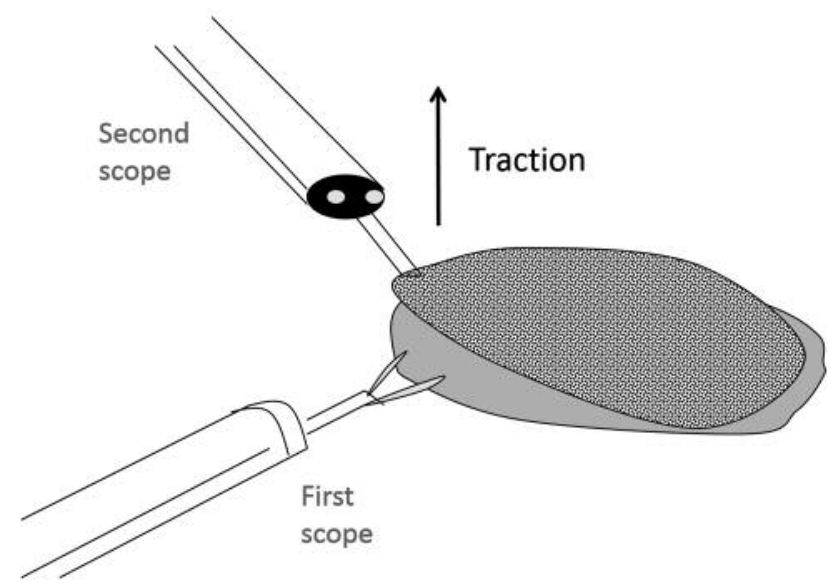

Figure 1. Schema of DEILO procedure. The second endoscope is inserted into the stomach, and the edge of the lesion is grasped and lifted with the grasping forceps.

(D-201-11804; Olympus). The second endoscope was a GIF-XP240 (Olympus), with an outer diameter of $7.7 \mathrm{~mm}$. An indigo carmine solution was sprayed onto the mucosa, and the mucosal surface surrounding the margin of the lesion was carefully marked using the tip of an electrosurgical hook knife (KD 620LR; Olympus) with electrocautery and a Q260J endoscope. Regions $2 \mathrm{~mm}$ from the margin of the lesion were marked, and $0.4 \%$ hyaluronic acid (MucoUp; Johnson \& Johnson, Tokyo, Japan) mixed with epinephrine and indigo carmine was injected into the submucosa to elevate the lesion. A circumferential mucosal incision was made around the lesion using an insulation-tipped (IT) knife 2 (Olympus) or a hook knife. Submucosal dissection was performed adequately with the Q260J scope at the portion of tissue grasped by forceps.

The XP240 endoscope was inserted into the stomach, and the edge of the lesion was elevated using grasping forceps (FG-4L-1; Olympus). This traction provided a clear view of the cutting plane and the tissue to be dissected (Figures 1 and 2). The resected specimen was retrieved using grasping forceps inserted from the XP240 endoscope. The important techniques required for DEILO include grasping and pulling the tissue with the forceps, and then excising the mucosa using a hook knife or an IT knife 2 and hemostatic forceps (FD-410LR; Coagrasper, Olympus).

Histological assessment of the resected specimen. The resected specimen was spread and fastened to a rubber plate using fine needles and then bathed in $10 \%$ formalin solution. Each specimen was cut into $2-\mathrm{mm}$ slices according to the standards of the Japanese Classification of Gastric Carcinoma (7). Each slice was processed for histopathological assessment of the histological characteristics of the tumor, depth of invasion, presence or absence of lymphatic or venous invasion, and completeness of resection.

\section{Results}

For 119 out of the $122(97.5 \%)$ tumors, the DEILO procedure was performed successfully, and en bloc resection was achieved. Three lesions could not be resected en bloc 

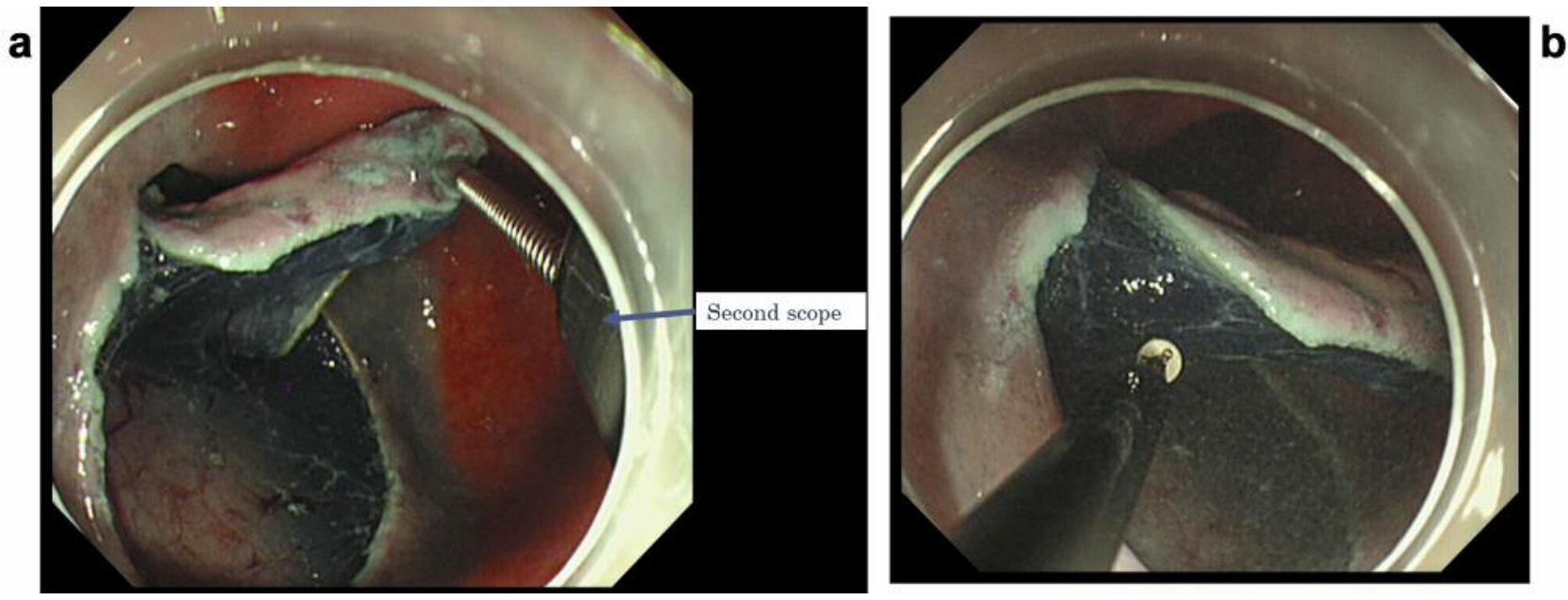

Figure 2. Intraoperative findings. a: The submucosa can be seen after lifting the mucosa with the grasping forceps, which were passed through the biopsy channel of the second endoscope. $b$ : The submucosal layer is dissected under direct visualization.

because of ulceration or tumor location, two on the lesser curvature in the upper portion of the stomach, and one the anterior wall in the middle portion of the stomach. The mean operative time for DEILO was 70.9 (range=20-207) minutes. The mean length of the postoperative hospital stay was 4 days. Three patients $(2.5 \%)$ sustained postoperative hemorrhage at 5,6 , and $6.5 \mathrm{~h}$ after the DEILO procedure, and the bleeding was successfully treated by placement of metallic clips or coagulation of the bleeding vessels (Table II). The lesions of the patients sustaining hemorrhage were on the greater curvature and the posterior wall in the middle portion, and the lesser curvature in the upper portion of the gastric body. The rate of perforation from DEILO was 3.3\% (4/122). All patients sustaining perforation were successfully treated nonsurgically using a combination of fasting, nasogastric tube drainage, proton pump inhibitors, and broad-spectrum antibiotics (Table II). Emergency surgery was not needed in any case.

The assessment of histological curability is summarized in Table II. Histopathologically, curative resection was achieved for 106 lesions (86.9\%). The median follow-up period was 24 months. Two patients were found to have local recurrence 15 and 19 months after DEILO, respectively. The resections performed for these patients were histologically incomplete.

\section{Discussion}

ESD is a safe, convenient, effective, and minimally invasive technique. However, ESD is technically difficult for large lesions. Because standard ESD is performed under direct vision using a cutting knife, the endoscopist's vision is obscured near the end of the procedure, when only a thin rim
Table II. Clinical outcomes for all patients included in the study

\begin{tabular}{lc}
\hline & Cases $(\mathrm{n}=122)$ \\
\hline En bloc resection, n (\%) & $119(97.5 \%)$ \\
Median treatment time (range), min & $70.9(20-207)$ \\
Mean postoperative hospital stay (range), days & $4(1-10)$ \\
Complications, $\mathrm{n}(\%)$ & \\
$\quad$ Postoperative bleeding & $3(2.5 \%)$ \\
Perforation & $4(3.3 \%)$ \\
Pneumonia & $0(0 \%)$ \\
Histological assessment of curability, n (\%) & \\
$\quad$ Curative resection & $106(86.9 \%)$ \\
$\quad$ Non-curative resection & $16(13.1 \%)$ \\
Local recurrence, $\mathrm{n}(\%)$ & $2(1.6 \%)$ \\
\hline
\end{tabular}

is attached to the submucosa. In addition, the dissected mucosa frequently cannot be stabilized; thus, the cutting line and inadvertent cutting of the submucosal layer may not be identified accurately. Although many types of ESD methods with traction have been reported, each method has its advantages and disadvantages (8).

The double scope method. We reported the effectiveness and feasibility of DEILO in 2004 and $2010(5,6)$. DEILO was developed to enable the use of two hands. DEILO refers to intraluminal surgery performed in the esophagus and stomach using two endoscopes inserted through the oral cavity. One benefit of DEILO is that the mucosal layer can be easily grasped and lifted using the grasping forceps to cut and directly visualize the submucosal layer. Double endoscopy allows the operator to apply the appropriate amount of 
tension to the submucosa to facilitate dissection and shortens the operating time. The technique can be applied to lesions in different areas of the stomach, and traction can be added easily, as needed. Furthermore, this procedure also enables easy releasing and regrasping of the lesion with the forceps if the traction is not sufficient. However, this method requires two light sources, two endoscopists, and two assistants.

Several other methods using double endoscopes have also been reported. Morita et al. described a double endoscope method, which required two light sources and an overtube with two channels to prevent interaction between the two endoscopes (9). Higuchi et al. reported another method, which required only one light source that could be transferred between two endoscopes, eliminating the problem of optical interference (10). Ahn et al. reported a thin trans-nasal endoscope-assisted ESD procedure. They reported that nasal bleeding due to trans-nasal access occurred too easily (11).

The double channel scope method. Yonezawa et al. (12), Neuhaus et al. (13), and Lee et al. (14) reported ESD using an R-scope, which has two movable instrument channels: one moving a pair of grasping forceps vertically for lesions with traction, and the other swinging a cutting device horizontally for dissection. Since the grasping forceps are inserted through the endoscope, they move synchronously with the endoscope, sometimes making it difficult to control the traction direction. Furthermore, a double-channel scope is thicker, heavier, and more difficult to manipulate than a single-channel scope.

The clip with line method. Oyama et al. (15), Jeon et al. (16), Li et al. (17), and Ota et al. (18) reported the clip with line method, which is a simple, easy, and useful method for achieving traction. A long silk line is tied to the clip, the submucosal side of the lesion is grasped and the line is pulled. This method creates a clear field of vision. However, the direction of traction is limited.

The magnetic anchor method. Kobayashi et al. (19) and Gotoda et al. (20) reported a magnetic anchor method. A magnetic anchor with magnetic weight and microforceps is placed at the edge of the lesion. Dissection is performed with suitable traction by using a high-power electromagnet placed outside the body. However, this method requires large and expensive instruments and systems.

The percutaneous traction method. Kondo et al. described percutaneous traction-assisted EMR (21), which requires a laparoscopic port with a trocar. A small snare is inserted into the gastric lumen through a port to grasp and pull the lesion. von Delius et al. reported on similar methods using a percutaneous endoscopic gastrostomy (PEG) minitrocar (22). The wire, which was fixed on the edge of the resected lesion, was pulled out through the PEG and applied tension on the submucosa.
Nishiwaki et al. reported transgastrostomic endoscopy-assisted ESD (23). A small-caliber endoscope was inserted through the mature gastrostomy, and the edge of the resecting lesion was grasped to apply the traction. However, these methods cannot be used for lesions on the anterior wall of the stomach.

External grasping forceps method. Imaeda et al. reported ESD using external grasping forceps (24, 25). External grasping forceps are used under the control of an endoscope and a second pair of grasping forceps. This method does not require any assistant to hold the forceps because the handle is locked. One endoscopist can therefore independently move the endoscope and forceps. Moreover, this procedure can also enable the release and regrasping of the lesion with the forceps. However, this method is sometimes difficult for lesions in the cardia and the lesser curvature or posterior wall of the upper gastric body.

Some methods are limited to lesions in certain areas. Our DEILO technique enables the endoscopist to visualize the submucosal layer directly for any stomach lesion (5). However, lesions near the gastric cardia were difficult to resect using DEILO, because the two endoscopes obstructed each other when the distal end of the bending section was turned. In addition, DEILO is more expensive than standard ESD because it requires two endoscopes, two light sources, and two endoscopists. One of the major benefits of DEILO is that it enables visual confirmation that the lesion is accurately and safely targeted because the submucosal layer is raised before being cut. The most effective method of preventing complications such as perforation and bleeding is to lift the submucosal layer and incise the target tissue under direct vision. In our study, the rates of perforation and bleeding were $3.3 \%$ and $2.5 \%$. Several studies have reported postoperative bleeding in $4 \%$ to $10 \%$ of patients undergoing ESD, and perforation in $1 \%$ to $5 \%$ of patients during ESD (26-28). The results of our study indicate that DEILO was effective and feasible compared to other methods with regard to complication rates.

In conclusion, DEILO is a good option for endoscopic treatment of EGC, because it is safe and technically easy for surgeons and endoscopists to perform this procedure using their left hands.

\section{Conflicts of Interest}

None.

\section{References}

1 Japanese Gastric Cancer Association: Japanese gastric cancer treatment guidelines 2010 (ver. 3). Gastric Cancer 14: 113-123, 2011.

2 Gotoda T, Yanagisawa A, Sasako M, Ono H, Nakanishi Y, Shimoda $\mathrm{T}$ and Kato $\mathrm{Y}$ : Incidence of lymph node metastasis from early gastric cancer: estimation with a large number of cases at two large centers. Gastric Cancer 3: 219-225, 2000. 
3 Ono H, Kondo H, Gotoda T, Shirao K, Yamaguchi H, Saito D, Hosokawa K, Shimoda T and Yoshida S: Endoscopic mucosal resection for treatment of early gastric cancer. Gut 48: 225-229, 2001.

4 Ono H, Hasuike N, Inui T, Takizawa K, Ikehara H, Yamaguchi $\mathrm{Y}$, Otake $\mathrm{Y}$ and Matsubayashi $\mathrm{H}$ : Usefulness of a novel electrosurgical knife, the insulation-tipped diathermic knife-2, for endoscopic submucosal dissection of early gastric cancer. Gastric Cancer 11: 47-52, 2008.

5 Kuwano H, Mochiki E, Asao T, Kato H, Shimura T and Tsutsumi S: Double endoscopic intralumenal operation for upper digestive tract diseases: proposal of a novel procedure. Ann Surg 239: 22-27, 2004

6 Mochiki E, Yanai M, Toyomasu Y, Ogata K, Andoh H, Ohno T, Aihara R, Asao T and Kuwano H: Clinical outcomes of double endoscopic intralumenal surgery for early gastric cancer. Surg Endosc 24: 631-636, 2010.

7 Japanese Gastric Cancer Association. Japanese Classification of Gastric Carcinoma: 3rd English edition. Gastric Cancer 14: 101112,2011

8 Imaeda H, Hosoe N, Kashiwagi K, Ohmori T, Yahagi N, Kanai $\mathrm{T}$ and Ogata H: Advanced endoscopic submucosal dissection with traction. World J Gastrointest Endosc 16: 286-295, 2014.

9 Morita Y, Masuda M, Tanaka S, Fujiwara M, Wakahara C, Toyonaga T and Azuma T: A new approach to treating difficult cases of early gastric cancer: development of a double scopeESD using transnasal endoscope with a "Split Barrel". Endoscopia Digestiva 22: 846-852, 2010 (in Japanese, English abstract)

10 Higuchi K, Tanabe S, Azuma M, Sasaki T, Katada C, Ishido K, Naruke A, Mikami T and Koizumi W: Double-endoscope endoscopic submucosal dissection for the treatment of early gastric cancer accompanied by an ulcer scar (with video). Gastrointest Endosc 78: 266-273, 2013.

11 Ahn JY, Choi KD, Choi JY, Kim MY, Lee JH, Choi KS, Kim DH, Song HJ, Lee GH and Jung HY: Transnasal endoscopeassisted endoscopic submucosal dissection for gastric adenoma and early gastric cancer in the pyloric area: a case series Endoscopy 43: 233-235, 2011.

12 Yonezawa J, Kaise M, Sumiyama K, Goda K, Arakawa H and Tajiri H: A novel double-channel therapeutic endoscope ("Rscope") facilitates endoscopic submucosal dissection of superficial gastric neoplasms. Endoscopy 38: 1011-1015, 2006.

13 Neuhaus H, Costamagna G, Devière J, Fockens P, Ponchon T and Rösch T: Endoscopic submucosal dissection (ESD) of early neoplastic gastric lesions using a new double-channel endoscope (the "R-scope"). Endoscopy 38: 1016-1023, 2006.

14 Lee SH, Gromski MA, Derevianko A, Jones DB, Pleskow DK, Sawhney M, Chuttani R and Matthes K: Efficacy of a prototype endoscope with two deflecting working channels for endoscopic submucosal dissection: a prospective, comparative, ex vivo study. Gastrointest Endosc 72: 155-160, 2010.

15 Oyama T, Kikuchi Y, Shimaya S, Tomori A, Hotta K, Miyata Y and Yamada S. Endoscopic mucosal resection using a hooking knife (hooking EMR). Stomach Intest 37: 1155-1161, 2002.

16 Jeon WJ, You IY, Chae HB, Park SM and Youn SJ: A new technique for gastric endoscopic submucosal dissection: peroral traction-assisted endoscopic submucosal dissection. Gastrointest Endosc 69: 29-33, 2009.
$17 \mathrm{Li} \mathrm{CH}$, Chen PJ, Chu HC, Huang TY, Shih YL, Chang WK and Hsieh TY: Endoscopic submucosal dissection with the pulley method for early-stage gastric cancer (with video). Gastrointest Endosc 73: 163-167, 2011.

18 Ota M, Nakamura T, Hayashi K, Ohki T, Narumiya K, Sato T, Shirai Y, Kudo K and Yamamoto M: Usefulness of clip traction in the early phase of esophageal endoscopic submucosal dissection. Dig Endosc 24: 315-318, 2012.

19 Kobayashi T, Gotohda T, Tamakawa K, Ueda H and Kakizoe T: Magnetic anchor for more effective endoscopic mucosal resection. Jpn J Clin Oncol 34: 118-123, 2004.

20 Gotoda T, Oda I, Tamakawa K, Ueda H, Kobayashi $\mathrm{T}$ and Kakizoe T: Prospective clinical trial of magnetic-anchor-guided endoscopic submucosal dissection for large early gastric cancer (with videos). Gastrointest Endosc 69: 10-15, 2009.

21 Kondo H, Gotoda T, Ono H, Oda I, Kozu T, Fujishiro M, Saito D and Yoshida S: Percutaneous traction-assisted EMR by using an insulation-tipped electrosurgical knife for early stage gastric cancer. Gastrointest Endosc 59: 284-288, 2004.

22 von Delius S, Karagianni A, von Weyhern $\mathrm{CH}$, Feussner $\mathrm{H}$, Schuster T, Schmid RM and Frimberger E: Percutaneously assisted endoscopic surgery using a new PEG-minitrocar for advanced endoscopic submucosal dissection (with videos). Gastrointest Endosc 68: 365-369, 2008.

23 Nishiwaki S, Araki H, Shirakami Y, Niwa Y, Iwashita M, Hatakeyama $\mathrm{H}$ and Saitoh K: Transgastrostomic endoscopyassisted endoscopic submucosal dissection. Endoscopy 41: E13, 2009.

24 Imaeda H, Iwao Y, Ogata H, Ichikawa H, Mori M, Hosoe N, Masaoka T, Nakashita M, Suzuki H and Inoue N: A new technique for endoscopic submucosal dissection for early gastric cancer using an external grasping forceps. Endoscopy 38: 1007$1010,2006$.

25 Imaeda H, Hosoe N, Ida Y, Kashiwagi K, Morohoshi Y, Suganuma K, Nagakubo S, Komatsu K, Suzuki H and Saito Y: Novel technique of endoscopic submucosal dissection using an external grasping forceps for superficial gastric neoplasia. Dig Endosc 21: 122-127, 2009.

26 Gotoda T: Endoscopic resection of early gastric cancer. Gastric Cancer 10: 1-11, 2007.

27 Choi MK, Kim GH, Park do Y, Song GA, Kim DU, Ryu DY, Lee BE, Cheong JH and Cho M: Long-term outcomes of endoscopic submucosal dissection for early gastric cancer: a single-center experience. Surg Endosc 27: 4250-4258, 2013.

28 Kosaka T, Endo M, Toya Y, Abiko Y, Kudara N, Inomata M, Chiba T, Takikawa Y, Suzuki K and Sugai T: Long-term outcomes of endoscopic submucosal dissection for early gastric cancer: A single-center retrospective study. Dig Endosc 26: 183191, 2014.
Received November 2, 2016

Revised November 24, 2016

Accepted November 29, 2016 